Artículo

\title{
Caracterización morfológica de accesiones de Moringa oleífera provenientes del sur-sureste de México
}

\author{
Rafael Ruiz Hernández ${ }^{1}$ \\ Arturo Pérez-Vázquez ${ }^{1 \S}$ \\ Eliseo García Pérez ${ }^{1}$ \\ Cesáreo Landeros Sánchez ${ }^{1}$ \\ Fredy Morales Trejo ${ }^{2}$ \\ Ramón Marcos Soto Hernández ${ }^{3}$ \\ ${ }^{1}$ Campus Veracruz-Colegio de Postgraduados. Carretera Xalapa-Veracruz km 88.5, Predio Tepetates, \\ Manlio F. Altamirano, Veracruz. CP. 91700. Tel. 229 2010770. (rafael.ruiz@colpos.mx; \\ geliseo@colpos.mx; clandero@ colpos.mx). ${ }^{2}$ Universidad Autónoma Chapingo. Carretera. México-Texcoco \\ km 38.5, Chapingo, Texcoco, Estado de México. CP. 56230. Tel. 5959521540. \\ (fredy.morales@colpos.mx). ${ }^{3}$ Colegio de Postgraduados. Carretera México-Texcoco km 36.5, Montecillo, \\ Texcoco, Estado de México. CP. 56230. Tel. 595 9570887. (msoto@colpos.mx). \\ ${ }^{\S}$ Autor para correspondencia: parturo@colpos.mx.
}

\section{Resumen}

La Moringa oleifera es una planta con gran capacidad de adaptación a diferentes condiciones edafoclimáticas. Los factores ambientales influyen en la morfología y fenología de esta especie. El objetivo fue caracterizar la morfología de 20 accesiones de Moringa oleifera provenientes del sursureste de México. Las semillas fueron recolectadas en cultivos comerciales de los estados de Veracruz, Oaxaca, Guerrero, Chiapas y Yucatán. Las semillas fueron sembradas en contenedores y se trasplantaron dos meses después de su germinación en campo con un diseño de bloques completamente al azar (DBCA). Cada siete días se registraron las variables cuantitativas y a los 301 días después del trasplante se evaluaron los descriptores morfológicos. Se observó que la accesión C2 presentó el mayor crecimiento $(273 \mathrm{~cm})$. La accesión Y2 presentó un diámetro de $43.22 \mathrm{~mm}$ y la accesión Y3 presentó 54 hojas. Se encontró una alta variación en el tamaño de la hoja, color de la flor y tallo e inicio de floración. El análisis de componentes principales identificó tres grupos. El análisis de componentes principales (PCA) mostró que los primeros cinco componentes explican $99.21 \%$ de la variación total y que los componentes 1 (52.87\%) y 2 (37.54\%) contribuyen con $90.41 \%$. Del análisis de conglomerados resultaron tres grupos con 0.76 de similitud, basado en la similitud euclidiana. La diferenciación morfológica de los diversos accesos de moringa permitió corroborar diferenciación varietal y la necesidad de instrumentar un programa genético de conservación, selección y fitomejoramiento de moringa en el sur-sureste de México.

Palabras clave: accesiones, agroecosistemas, fenotipos, moringa.

Recibido: agosto de 2021

Aceptado: octubre de 2021 


\section{Introducción}

Las plantas tienen, en general, la capacidad de adaptarse a las diversas condiciones que existen en el planeta. Este ajuste fisiológico les permite sobrevivir ante climas diversos y adversos. Sin embargo, los factores externos influyen en el crecimiento y la producción a través de la influencia directa en sus procesos fisiológicos y bioquímicos (Tesfay et al., 2011; Santiago y Bezerra, 2017). Moringa oleifera Lam., es una especie vegetal perenne, de rápido crecimiento que presenta gran plasticidad agroecológica (Pérez et al., 2010). La importancia mundial de esta planta radica en su uso para mejorar contribuir al mejoramiento de la nutrición y salud humana, a garantizar la seguridad alimentaria, promover el desarrollo económico en zonas rurales y a mitigar los efectos del cambio climático (NRC, 2006).

Todas las partes de la moringa presentan principios bioactivos de importancia alimenticia y medicinal (Martín et al., 2013). Además, presenta una alta capacidad de almacenamiento de compuestos activos que está determinada por la variedad o por la modificación que haya sufrido la accesión en el ambiente recolectado (Baiyeri et al., 2015). En la India, se han identificado las variedades de moringa: PKM-1, PKM-2, Jaffna, Chavakacheri Murungai, Chemmurungai, Kaadumurungai palmurungai, Puna murungai y Kodikkal murungai que muestran diferencias fenológicas y morfológicas.

La caracterización morfológica es una herramienta fundamental para la selección, conservación, mejora y creación de nuevas variedades (Popoola et al., 2016; Kumar et al., 2017). El estudio de accesiones en condiciones ambientales homogéneas permite detectar la variabilidad en el crecimiento, floración, número y tamaño de hojas y frutos y permite identificar la resistencia a diversos tipos de estrés ambiental (Resmi et al., 2005). A pesar de la gran adaptabilidad que posee la planta de moringa, se han encontrado poblaciones caducifolias en climas subtropicales (Folkard et al., 1999).

El conocimiento de la diversidad morfológica en moringa puede convertirse en un recurso para su mejoramiento a través de la sección de variedades élite adaptadas a las condiciones locales (Leone et al., 2015). El Sur-Sureste de México, cuenta con diversas plantaciones de moringa. Sin embargo, no existe información detallada de la variación morfológica de los diversos accesos. La variación morfológica en cualquier planta puede atribuirse a factores edafoclimáticos, genéticos, de manejo agronómico o a la su combinación de éstos (Chaves-Bedoya et al., 2017). El objetivo fue caracterizar la morfología de accesiones de M. oleifera provenientes del Sursureste de México.

\section{Materiales y métodos}

\section{Área de estudio}

La investigación fue en la región sur-sureste de México por la presencia de cultivos comerciales de moringa y esta característica favorecía la disponibilidad de semilla. Las semillas de los accesos estudiados provienen de Veracruz, Oaxaca, Guerrero, Chiapas y Yucatán (Cuadro 1, Figura 1). La investigación se realizó en el Colegio de Postgraduados, Campus Veracruz, ubicado geográficamente a los $19^{\circ} 16^{\prime} 32^{\prime \prime}$ de latitud norte, $96^{\circ} 16^{\prime} 32^{\prime \prime}$ de longitud oeste, a una altitud de $16 \mathrm{~m}$ (Valdés et al., 2014). El clima es cálido subhúmedo $\left(\mathrm{AW}_{0}\right)$, con una precipitación anual acumulada de $1000 \mathrm{~mm}$ y una temperatura media anual de $27^{\circ} \mathrm{C}$ (Olguín, 1999). 
Cuadro 1. Procedencia de las semillas de $M$. oleifera del sur-sureste de México.

\begin{tabular}{|c|c|c|c|c|c|c|}
\hline Núm. & Estado & Accesión & Municipio & Comunidad & Longitud & Latitud \\
\hline 1 & Veracruz & $\mathrm{V} 1$ & Soledad de Doblado & El Progreso & -96.4022719 & 19.0818742 \\
\hline 2 & Veracruz & $\mathrm{V} 2$ & Paso del Macho & Loma Pelada & -96.5398368 & 18.9258796 \\
\hline 3 & Veracruz & V3 & Tierra Blanca & Colonia Pemex & -96.3429545 & 18.435 \\
\hline 4 & Veracruz & V4 & Misantla & Santa Cruz Hidalgo & -96.8628092 & 19.9555656 \\
\hline 5 & Oaxaca & $\mathrm{O} 1$ & $\begin{array}{l}\text { Santa Cruz } \\
\text { Xoxocotlán }\end{array}$ & $\begin{array}{c}\text { San Juan Bautista La } \\
\text { Raya }\end{array}$ & -96.7280556 & 16.9791667 \\
\hline 6 & Oaxaca & $\mathrm{O} 2$ & Santa María Huatulco & La Herradura & -96.3658333 & 15.7772222 \\
\hline 7 & Oaxaca & $\mathrm{O} 3$ & Mariscala de Juárez & Guadalupe la Huertilla & -98.1088889 & 17.8513889 \\
\hline 8 & Oaxaca & $\mathrm{O} 4$ & Tuxtepec & San Juan Bautista & -96.1286697 & 18.087694 \\
\hline 9 & Guerrero & G1 & Acapulco de Juárez & Bejuco & -99.6977778 & 16.8216667 \\
\hline 10 & Guerrero & $\mathrm{G} 2$ & Acapulco de Juárez & Parotillas & -99.61558371 & 16.8787834 \\
\hline 11 & Guerrero & G3 & Acapulco de Juárez & Concepción & -99.66028879 & 16.8799601 \\
\hline 12 & Guerrero & G4 & Tecpan de Galeana & Mitla & -99.89343517 & 16.8789425 \\
\hline 13 & Chiapas & $\mathrm{C} 1$ & Tuzantán & Villa Hidalgo & -92.374722 & 15.108056 \\
\hline 14 & Chiapas & $\mathrm{C} 2$ & Tuxtla Gutiérrez & Colonia La Salle & -93.0868889 & 16.7429445 \\
\hline 15 & Chiapas & $\mathrm{C} 3$ & Tuxtla Gutiérrez & Santa Cruz & -93.108986 & 16.783481 \\
\hline 16 & Chiapas & $\mathrm{C} 4$ & Tuxtla Gutiérrez & San Juan & -93.103645 & 16.747307 \\
\hline 17 & Yucatán & Y1 & Tzucacah & Tzucacah & -89.0391111 & 20.0720278 \\
\hline 18 & Yucatán & Y2 & Mérida & Frac. el Parque & -89.5872222 & 20.9711111 \\
\hline 19 & Yucatán & Y3 & Peto & Teshan & -88.62125 & 20.1486389 \\
\hline 20 & Yucatán & Y4 & Baca & Felipe Carrillo Puerto & -89.6070099 & 20.9954688 \\
\hline
\end{tabular}

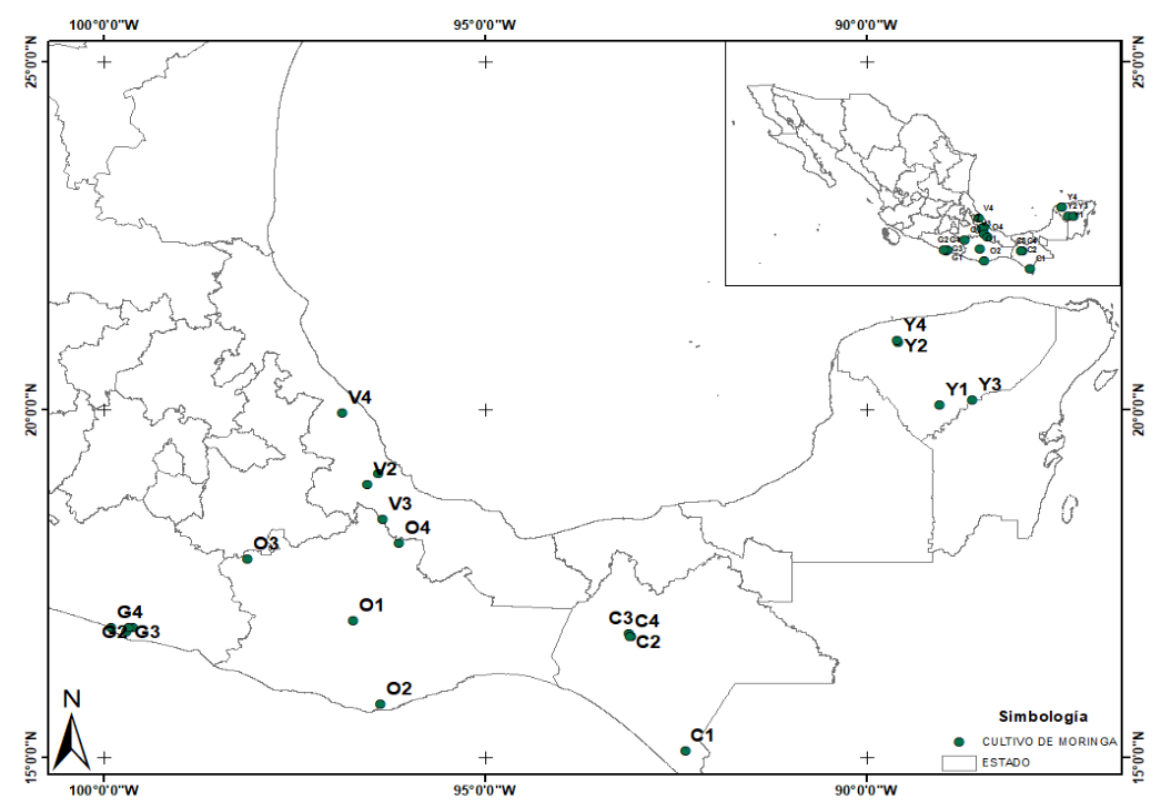

Figura 1. Ubicación geográfica de los puntos de recolecta de $M$. oleifera en el Sursureste de México. 


\section{Material biológico}

Se recolectaron semillas de moringa de diferentes cultivos comerciales en el sur-sureste de México durante los meses de febrero a mayo de 2018. Se seleccionaron 30 semillas sanas de cada punto de recolecta y se midieron las variables de longitud, grosor y peso de la semilla. Se evaluaron 20 accesiones, mismas que se muestran en el (Cuadro 2).

Cuadro 2. Variables morfológicas de los accesos de moringa del sur-sureste de México.

\begin{tabular}{ccccc}
\hline Núm. & Accesión & $\begin{array}{c}\text { Peso promedio }(\mathrm{mg}) \\
\pm \text { error estándar }\end{array}$ & $\begin{array}{c}\text { Largo promedio }(\mathrm{mm}) \\
\pm \text { error estándar }\end{array}$ & $\begin{array}{c}\text { Grosor promedio (diámetro) } \\
(\mathrm{mm}) \pm \text { error estándar }\end{array}$ \\
\hline 1 & V1 & $364.33^{* *} \pm 8.19$ & $11.93 \pm 0.26$ & $8.33 \pm 0.14$ \\
2 & V2 & $296.67 \pm 11.31$ & $9.5 \pm 0.24$ & $7.33 \pm 0.18$ \\
3 & V3 & $325.33 \pm 10.5$ & $8.93 \pm 0.18$ & $8.5 \pm 0.18$ \\
4 & V4 & $331.43 \pm 4.59$ & $9.57 \pm 0.2$ & $10.43 \pm 0.3$ \\
5 & O1 & $332.33 \pm 6.91$ & $11.67 \pm 0.23$ & $9.5 \pm 0.13$ \\
6 & O2 & $379.67 \pm 8.2$ & $11.33 \pm 0.19$ & $10.63 \pm 0.15$ \\
7 & O3 & $324.67 \pm 6.19$ & $13.53 \pm 0.25$ & $10.77 \pm 0.09$ \\
8 & O4 & $364.67 \pm 8.63$ & $11.87 \pm 0.19$ & $11.37 \pm 0.15$ \\
9 & G1 & $309.67 \pm 9.83$ & $12.43 \pm 0.23$ & $9.53 \pm 0.13$ \\
10 & G2 & $356 \pm 7.99$ & $13.97 \pm 0.33$ & $10 \pm 0.16$ \\
11 & G3 & $408.67 \pm 10.42$ & $12.97 \pm 0.28$ & $11.07 \pm 0.13$ \\
12 & G4 & $580.67 \pm 2.05$ & $14.2 \pm 0.18$ & $12.3 \pm 0.12$ \\
13 & C1 & $485.33 \pm 10.36$ & $14.9 \pm 0.35$ & $10.93 \pm 0.21$ \\
14 & C2 & $461 \pm 10.3$ & $13.8 \pm 0.24$ & $11.83 \pm 0.14$ \\
15 & C3 & $480 \pm 8.04$ & $13.87 \pm 0.25$ & $11.97 \pm 0.14$ \\
16 & C4 & $308.33 \pm 7.08$ & $12.13 \pm 0.24$ & $9.53 \pm 0.22$ \\
17 & Y1 & $337.67 \pm 9.38$ & $13.03 \pm 0.23$ & $11.2 \pm 0.18$ \\
18 & Y2 & $460 \pm 1.25$ & $14.3 \pm 0.35$ & $10.6 \pm 0.19$ \\
19 & Y3 & $349.67 \pm 8.7$ & $12.47 \pm 0.22$ & $10.37 \pm 0.21$ \\
20 & Y4 & $428.67 \pm 12.76$ & $13.6 \pm 0.27$ & $11.27 \pm 0.23$ \\
\hline V= Veracruz; O= Oaxaca; G $=$ Guerrero; C Chiapas; Y= Yucatán; $*$ valor promedio de 30 semillas.
\end{tabular}

\section{Siembra y trasplante}

Las semillas fueron sembradas en bolsas de vivero de color negro de 27 x $27 \mathrm{~cm}$. El sustrato utilizado estuvo compuesto por suelo, estiércol-lombricomposta y arena (5:4:1). Después de 15 días la germinación se seleccionó las plántulas de mayor vigor y de un tamaño similar y dos meses después, se trasplantaron en campo. El tipo de suelo en campo era franco-arcilloso.

\section{Tratamientos y diseño experimental}

El diseño experimental fue de bloques completamente al azar con cinco repeticiones. El tamaño de la superficie utilizada fue de $900 \mathrm{~m}^{2}$. La distancia entre individuos y surcos fue de $3 \mathrm{~m}$. Cada siete días se registraron las variables de altura, diámetro basal (a $10 \mathrm{~cm}$ del suelo), número de ramas y hojas. Diariamente se aplicaron $2 \mathrm{~L}$ de agua a cada planta a través del riego por goteo. 


\section{Caracterización morfológica}

Para la caracterización morfológica se utilizaron descriptores cuantitativos y cualitativos para cada órgano de la planta. Los cuantitativos registrados fueron: altura, diámetro del tallo, número de hojas, número de ramas, número de flores, longitud de la hoja, ancho de la hoja, longitud del peciolo, longitud del foliolo, ancho del foliolo y días a la primera floración. Para los descriptores cualitativos se utilizaron los publicados por Mgendi et al. (2011); Zhigila et al. (2015); Popoola et al. (2016). Los descriptores cualitativos fueron: color de peciolo de la hoja (1: verde claro, 2: verde, 3: violeta leve, 4: violeta medio, 5: violeta intenso), forma de la hoja (1: ovalo, 2: oblongo, 3: ovalo oblongo y 4: elíptica), ápice de la hoja (1: obtuso y 2: agudo), pubescencia en la hoja (0: ausente y 2: presente), color de la flor (1: blanco, 2: blanco-crema, 3: blanco-rosa, 4: blanco-crema-rosa y 5: rosáceo), manchas purpuras en las flores (0: ausente y 1: presente) y color de anteras (1: amarillas y 2: naranjas).

\section{Análisis estadísticos}

Los valores medios de los descriptores se evaluaron por estadísticas descriptivas. El coeficiente de correlación, los análisis de componentes principales y el análisis de conglomerado se realizaron a través de un agrupamiento jerárquico con coeficiente de la variación en el método del grupo de pares no ponderados (distancia euclidiana). El diagrama de dispersión bidimensional se realizó a través de la variación porcentual de los dos primeros análisis de componentes principales utilizando el programa PAST Versión 3.0.

\section{Resultados y discusión}

\section{Germinación}

El mayor porcentaje de germinación se obtuvo en la accesión G3 (100\%) y el menor porcentaje fue de 13\% para las accesiones C4, O1, G4, C1, C4 y Y1. Al momento del trasplante, la accesión V3 midió $108 \mathrm{~cm}$ siendo la mayor altura. Se observaron diferencias estadísticas significativas $(p<0.05)$ en la altura y diámetro del tallo de las 20 accesiones evaluadas. La accesión C3 presentó el diámetro mayor y el mayor número de hojas correspondió a la accesión O4 (Cuadro 3).

Cuadro 3. Variables registradas de Moringa oleifera al momento del trasplante.

\begin{tabular}{cccccc}
\hline \multirow{2}{*}{ Núm. } & Accesión & $\begin{array}{c}\text { Germinación } \\
(\%)\end{array}$ & $\begin{array}{c}\text { Altura }(\mathrm{cm}) \text { (media } \\
\pm \text { error estándar) }\end{array}$ & $\begin{array}{c}\text { Diámetro (mm) (media } \\
\pm \text { error estándar) }\end{array}$ & $\begin{array}{c}\text { Núm. de hojas (media } \\
\pm \text { error estándar) }\end{array}$ \\
\hline 1 & V1 & $60^{*}$ & $101.8 \pm 5.12 \mathrm{de}$ & $9 \pm 0.55 \mathrm{cdefgh}$ & $8.6 \pm 0.24 \mathrm{ab}$ \\
2 & $\mathrm{~V} 2$ & 13 & $98.6 \pm 5.82 \mathrm{de}$ & $8.2 \pm 0.37 \mathrm{bcdefg}$ & $7 \pm 0.32 \mathrm{a}$ \\
3 & $\mathrm{~V} 3$ & 50 & $108 \pm 7.35 \mathrm{e}$ & $10.25 \pm 0.48 \mathrm{gh}$ & $7.25 \pm 0.48 \mathrm{a}$ \\
4 & $\mathrm{~V} 4$ & 63 & $59.4 \pm 2.86 \mathrm{a}$ & $5.8 \pm 0.37 \mathrm{a}$ & $6.6 \pm 0.24 \mathrm{a}$ \\
5 & O1 & 13 & $98.6 \pm 3.52 \mathrm{de}$ & $8.6 \pm 0.4 \mathrm{cdefgh}$ & $7.4 \pm 0.24 \mathrm{ab}$ \\
6 & O2 & 10 & $85.8 \pm 2.29 \mathrm{cde}$ & $8 \pm 0.55 \mathrm{abcdef}$ & $7.8 \pm 0.2 \mathrm{ab}$ \\
7 & O3 & 43 & $84 \pm 4.89 \mathrm{bcd}$ & $9.4 \pm 0.68 \mathrm{defgh}$ & $6.6 \pm 0.24 \mathrm{a}$ \\
8 & O4 & 47 & $103.4 \pm 1.83 \mathrm{de}$ & $10 \pm 0.32 \mathrm{fgh}$ & $12.4 \pm 3.71 \mathrm{~b}$ \\
\hline
\end{tabular}




\begin{tabular}{cccccc}
\hline \multirow{2}{*}{ Núm. } & Accesión & $\begin{array}{c}\text { Germinación } \\
(\%)\end{array}$ & $\begin{array}{c}\text { Altura }(\mathrm{cm}) \text { (media } \\
\pm \text { error estándar) }\end{array}$ & $\begin{array}{c}\text { Diámetro (mm) (media } \\
\pm \text { error estándar) }\end{array}$ & $\begin{array}{c}\text { Núm. de hojas (media } \\
\pm \text { error estándar) }\end{array}$ \\
\hline 9 & $\mathrm{G} 1$ & 90 & $62 \pm 2.21 \mathrm{ab}$ & $6.2 \pm 0.37 \mathrm{ab}$ & $6.6 \pm 0.24 \mathrm{a}$ \\
10 & $\mathrm{G} 2$ & 33 & $84.4 \pm 3.26 \mathrm{bcd}$ & $7.2 \pm 0.2 \mathrm{abcd}$ & $7.2 \pm 0.2 \mathrm{a}$ \\
11 & $\mathrm{G} 3$ & 100 & $72.4 \pm 3.3 \mathrm{abc}$ & $7 \pm 0.32 \mathrm{abc}$ & $6 \pm 0.63 \mathrm{a}$ \\
12 & $\mathrm{G} 4$ & 13 & $95.8 \pm 2.94 \mathrm{de}$ & $7.6 \pm 0.24 \mathrm{abcde}$ & $7.8 \pm 0.37 \mathrm{ab}$ \\
13 & $\mathrm{C} 1$ & 13 & $85.8 \pm 3.07 \mathrm{cde}$ & $8.6 \pm 0.4 \mathrm{cdefgh}$ & $6.6 \pm 0.75 \mathrm{a}$ \\
14 & $\mathrm{C} 2$ & 50 & $82 \pm 1.64 \mathrm{abcd}$ & $7 \pm 0.55 \mathrm{abc}$ & $7.4 \pm 0.68 \mathrm{ab}$ \\
15 & $\mathrm{C} 3$ & 63 & $102.5 \pm 10.31 \mathrm{de}$ & $10.5 \pm 0.5 \mathrm{~h}$ & $9.75 \pm 0.25 \mathrm{ab}$ \\
16 & $\mathrm{C} 4$ & 13 & $86.75 \pm 3.35 \mathrm{cde}$ & $10 \pm 0.41 \mathrm{fgh}$ & $8.5 \pm 0.29 \mathrm{ab}$ \\
17 & $\mathrm{Y} 1$ & 13 & $93 \pm 2.94 \mathrm{cde}$ & $8.6 \pm 0.4 \mathrm{cdefgh}$ & $6.6 \pm 0.75 \mathrm{a}$ \\
18 & $\mathrm{Y} 2$ & 77 & $90 \pm 2.76 \mathrm{cde}$ & $10.2 \pm 0.37 \mathrm{fgh}$ & $7.2 \pm 0.37 \mathrm{a}$ \\
19 & Y3 & 60 & $96.6 \pm 4.35 \mathrm{de}$ & $9.6 \pm 0.4$ efgh & $8 \pm 0.32 \mathrm{ab}$ \\
20 & Y4 & 23 & $99.8 \pm 7.7 \mathrm{de}$ & $8.4 \pm 0.4 \mathrm{bcdefgh}$ & $8.2 \pm 0.2 \mathrm{ab}$ \\
\hline
\end{tabular}

$*$ * valor promedio de 10 plántulas. Medias con letras similares no presentan diferencias estadísticas significativas $(p>$ $0.05)$.

Se encontró gran variación en el porcentaje de germinación de las accesiones sembradas. Esta variación se atribuye a la calidad de la semilla y al tiempo de almacenaje antes de la siembra (Du Toit et al., 2017). Aunado a esto, al potencial genético y los factores ambientales como la temperatura, precipitación y la altitud influyen en el desarrollo de la semilla antes de la colecta y determinan su tamaño y peso (Baiyeri et al., 2015; Ledea-Rodríguez et al., 2018).

Este proceso afecta la viabilidad de semillas de más de un año de almacenadas en intervalos de temperatura de 23 a $25^{\circ} \mathrm{C}$. Por tanto, se recomienda que las semillas sean conservadas en las vainas (Fotouo et al., 2015). El promedio de días de germinación de las accesiones sembradas fue de 11. La geminación más temprana ocurrió a los 8 días y la más tardía a los 14 días. Este intervalo es similar a los 6 y 13 días reportado por Popoola et al. (2016); Zaku et al. (2015); mientras que Kumar et al. (2014) mencionaron que la germinación ocurre entre los 10 y 12 días después de la siembra. Ramos et al. (2010) reportaron que la germinación empieza a partir de los 8 días después de la siembra y a los 25 días aparecen las hojas primarias.

\begin{abstract}
Altura
Las accesiones C2 y G2 fueron las de mayor tamaño y alcanzaron una altura promedio de 273 y $271 \mathrm{~cm}$ respectivamente. La accesión Y4 presentó una altura promedio de $164 \mathrm{~cm}$, siendo el menor crecimiento registrado durante los 301 días después del trasplante (DDT) (Figura 2).

A los seis meses después del trasplante la altura mínima y máxima fueron 1.45 y $2.25 \mathrm{~m}$, respectivamente. Estos valores son inferiores a los 5.17 y $10.27 \mathrm{~m}$ reportados por Popoola et al. (2016). Esta menor talla se puede atribuir a la falta de precipitación, ya que el agua tiene un efecto directo en el crecimiento de la planta debido a que en condición de sequía disminuye la división y expansión celular (Taiz y Zeiger, 2009).
\end{abstract}




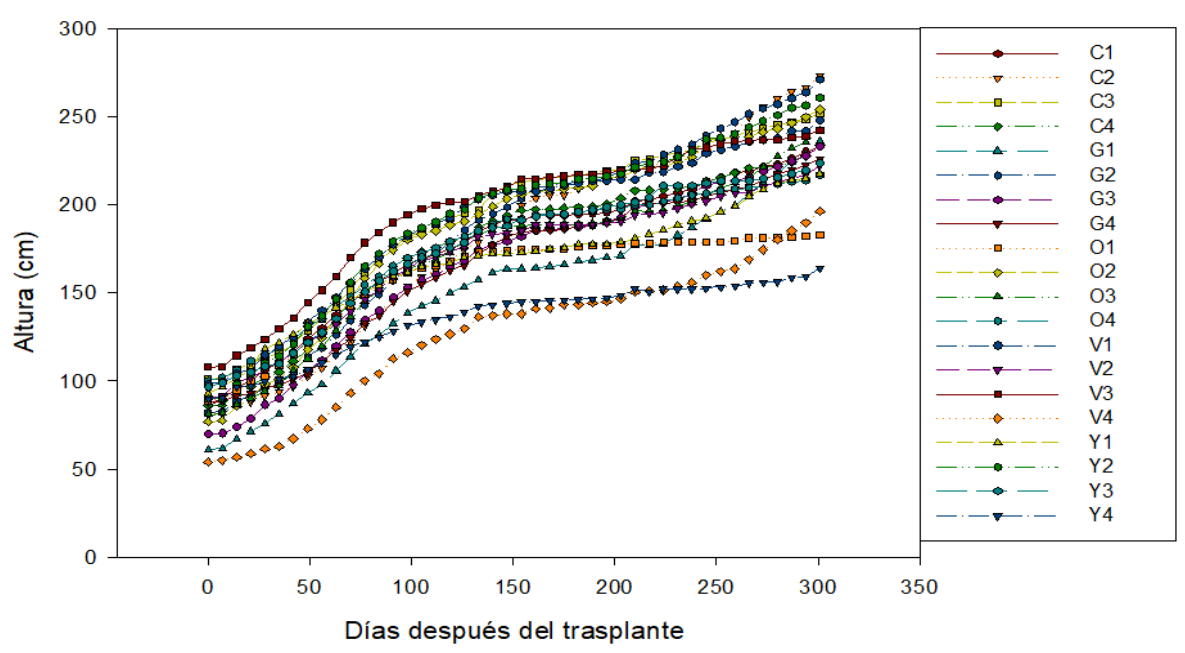

Figura 2. Altura de la planta de las 20 accesiones de Moringa oleifera recolectadas en el sur-sureste de México. Periodo: 0 al 301 DDT.

En relación con el número de ramas, se tuvieron pocas y estas emergieron después de los 2 m de altura. Popoola et al. (2016) menciona que la ramificación es moderada. Dao y Kabore (2015) registraron en moringa, a los dos meses de 8 a 15 ramas por árbol. En este trabajo se tuvo un crecimiento monopódico en las accesiones evaluadas.

\section{Diámetro del tallo}

El mayor diámetro a una altura de $10 \mathrm{~cm}$ del suelo se observó en la accesión Y2 con un valor promedio de $43.22 \mathrm{~mm}$ y el menor valor correspondió a la accesión Y4 con $25.61 \mathrm{~mm}$ a los 301 días después del trasplante. Los colores de tallo con mayor frecuencia fueron gris y gris blanquecino. Panshin y Zeeuw (1970) mencionan que el engrosamiento del tallo se debe a procesos relacionados en xilema y floema. Por tanto, pueden diferir entre accesiones. El engrosamiento del tallo y su morfología entre los ecotipos de moringa es diverso debido a la gran plasticidad que presenta (Förster et al., 2015).

\section{Hojas}

Después del trasplante se observó una defoliación en todas las accesiones. A los 301 DDT, la accesión Y3 presentó 54 hojas, siendo el valor más alto. La accesión O1 presentó 9 hojas, siendo la accesión con el menor número promedio de hojas. La longitud de la hoja más corta fue de 28.8 cm (Y3) y la más larga de $47 \mathrm{~cm}$ (C2). El ancho de la hoja fluctuó entre 16.8 (O1) y $38.33 \mathrm{~cm}(\mathrm{Y} 1)$. Se registró la presencia de pubescencias en las acciones G4, C3 y G2. Estos valores son superiores al rango de 21.4 a $54.2 \mathrm{~cm}$ de largo y de 10.1 a $41.6 \mathrm{~cm}$ del ancho reportado por Zhigila et al. (2015). Dao y Kabore (2015) reportaron longitudes promedio de hojas desde 16 hasta $44 \mathrm{~cm}$ y valores de 10.5 hasta $34 \mathrm{~cm}$ de ancho, con un número de pinas de 5 a 12 por hoja.

La longitud de la hoja está influenciada por la humedad relativa y la precipitación promedio anual. Las características fenotípicas se ven afectadas por los factores edáficos y dan como resultado cambios epigenéticos (Shahzad et al., 2013). Las hojas de moringa son consumidas por su alto valor nutricional (Förster et al., 2015). Por ello, el tamaño, color y número de hojas son importantes para la producción de biomasa, contenido nutricional y mejoramiento genético. 
También se identificó la presencia de pigmentación púrpura en el peciolo y raquis de las accesiones $\mathrm{C} 1, \mathrm{O} 2$ y Y2. El color oscuro en la hoja representa una mayor cantidad de clorofila y la existencia de este fotosintato promueve un mayor crecimiento (Opare-Obuobi, 2012).

La pigmentación violeta en el peciolo de hoja está determinada por el contenido de antocianinas. La presencia de antocianinas está determinada por las condiciones ambientales y puede ser de coloración purpura o rosada. Usualmente, los cultivos en áreas bajo sequía presentan mayor producción de antocianinas como mecanismo para evitar el estrés (Shahzad et al., 2013). Se notó la presencia de pubescencias en las hojas, brotes tiernos y filamentos de la antera. Existió defoliación ocasionada por las altas temperaturas y poca precipitación. Vasconcelos et al. (2019) mencionaron que el estrés hídrico ocasionado por la falta de precipitación influye en la morfología de la hoja y la fisiología de las plantas. En este trabajo se observó el efecto del estrés en la defoliación y amarillamiento de las hojas.

\section{Flores}

El inicio de la floración se registró contando los días desde la siembra de las accesiones. La accesión O4 fue la primera en iniciar el proceso de floración a los 129 días después de la siembra (dds). La última accesión en florecer fue la G3 a los 240 dds. Las accesiones Y2 y Y5 presentaron flores de color blanco rosáceo, la C2 y V3 blancas cremosas y la O1 flores blancas. Las manchas purpuras fueron identificadas en las accesiones G2 y V1 (Cuadro 4). La floración en las accesiones cultivadas comenzó a los 129 dds y se mantuvo durante los meses octubre-mayo. Sin embargo, la caída de flores impidió que muchas llegaran a la antesis limitando el proceso de polinización.

Cuadro 4. Valores de tendencia central para los descriptores cuantitativos de Moringa oleifera Lam.

\begin{tabular}{ccccccc}
\hline Núm. & Descriptor & Media & $\begin{array}{c}\text { Desviación } \\
\text { estándar }\end{array}$ & Máximo & Mínimo & Varianza \\
\hline 1 & Altura $(\mathrm{cm})$ & $231.45^{*}$ & 27.37 & $273(\mathrm{C} 2)$ & $164(\mathrm{Y} 4)$ & 749.32 \\
2 & Diámetro (mm) & 32.58 & 4.75 & $44(\mathrm{Y} 2)$ & $23.6(\mathrm{O} 1)$ & 22.59 \\
3 & Núm. hojas & 20.72 & 9.85 & $54(\mathrm{Y} 3)$ & $9.8(\mathrm{O} 1)$ & 97.09 \\
4 & Núm. ramas & 2.64 & 2.94 & $12.6(\mathrm{C} 2)$ & $0.4(\mathrm{O} 1)$ & 8.64 \\
5 & Núm. flores & 0.52 & 0.75 & $2.67(\mathrm{Y} 1)$ & $0(\mathrm{~V} 4, \mathrm{O} 1$ y O3) & 0.56 \\
6 & Núm. frutos & 0.35 & 0.43 & $1.6(\mathrm{O} 2)$ & $0(\mathrm{~V} 4, \mathrm{O} 1, \mathrm{O} 3$ y G1) & 0.19 \\
7 & Longitud de la hoja (cm) & 37.72 & 4.51 & $47(\mathrm{G} 1)$ & $28.8(\mathrm{Y} 3)$ & 20.33 \\
8 & Ancho de la hoja (cm) & 26.71 & 5.72 & $38.33(\mathrm{Y} 1)$ & $16.8(\mathrm{O} 1)$ & 32.72 \\
9 & Longitud del peciolo (cm) & 10.46 & 1.51 & $13(\mathrm{C} 1)$ & $7.6(\mathrm{Y} 3)$ & 2.27 \\
10 & Longitud del foliolo (mm) & 15.9 & 2.22 & $21.75(\mathrm{Y} 2)$ & $12(\mathrm{Y} 3)$ & 4.92 \\
11 & Ancho del foliolo (último) & 7.44 & 1.18 & $9.2(\mathrm{~V} 2)$ & $5(\mathrm{O} 3)$ & 1.4 \\
12 & Días a la primera floración & 180.53 & 28.89 & $240(\mathrm{G} 3)$ & $129.6(\mathrm{O} 4)$ & 834.51 \\
\hline${ }^{*}=$ valor promedio de cinco plantas. Valores obtenidos a los 301 DDT e inicio de la floración obtenido en DDS.
\end{tabular}


La floración en moringa puede ocurrir una vez o dos veces al año, dependiendo de las condiciones ambientales (temperatura y precipitación). Price (2000) mencionó que la floración puede ocurrir cuatro veces o durante el año. La pigmentación de las flores varió de blanco, blanco cremoso y blanco rosáceo. Algunas accesiones presentaron pigmentación púrpura en los pétalos. Popoola et al. (2016) registraron flores blancas con pigmentación purpura, blanco o blanco cremoso sin pigmentación y 50\% de la floración ocurrió en los 161 y 167 días. Moringa es una especie que presenta gran variabilidad en el color de sus flores y en algunas variedades de la India se han registrado flores con base rosa y rosa oscuro (PPV y FR, 2001).

Diversos estudios afirmaron que las flores de color rosa y rosa oscuro reciben más visitas de abejorros que las de color amarillo. Este factor representa, aparentemente, una estrategia de adaptación y reproducción (Bradshaw y Schemske, 2003; Reverté et al., 2016). Por tanto, se promueve la polinización cruzada entre accesiones de moringa y facilita la creación de nuevas variedades. Factores como la temperatura y la humedad del suelo influyen en el incremento de las flores, viabilidad del polen y disminuyen el número de frutos (Muhl et al., 2013).

\section{Coeficiente de correlación (r) de Pearson de los caracteres morfológicos}

La matriz de correlación de Pearson entre los descriptores cuantitativos de moringa se muestra en el Cuadro 5. Se encontró una correlación positiva entre el largo de la hoja con el ancho $(\mathrm{r}=0.857)$ y el largo de la hoja con el largo del peciolo $(r=0.851)$. También se encontró una correlación entre el diámetro y la altura $(\mathrm{r}=0.675)$. Se identificó una correlación negativa entre los días a la primera floración con el número de flores $(\mathrm{r}=-0.599)$ y con el número de frutos $(\mathrm{r}=-0.361)$.

Cuadro 5. Matriz de correlación de descriptores cuantitativos de Moringa oleifera a los 301 DDT.

\begin{tabular}{|c|c|c|c|c|c|c|c|c|c|c|c|c|}
\hline & $\begin{array}{l}\text { Altura I } \\
(\mathrm{cm})\end{array}$ & $\begin{array}{l}\text { Diámetro } \\
(\mathrm{mm})\end{array}$ & $\begin{array}{l}\text { Núm. } \\
\text { hojas }\end{array}$ & $\begin{array}{l}\text { Núm. } \\
\text { ramas }\end{array}$ & $\begin{array}{l}\text { Núm. } \\
\text { flores }\end{array}$ & $\begin{array}{l}\text { Núm. } \\
\text { frutos }\end{array}$ & $\begin{array}{l}\text { L. } \\
\text { hoja }\end{array}$ & A. hoja & $\begin{array}{c}\text { L. } \\
\text { peciolo }\end{array}$ & $\begin{array}{l}\mathrm{L} . \\
\text { foliolo }\end{array}$ & $\begin{array}{c}\text { A. } \\
\text { foliolo }\end{array}$ & $\begin{array}{c}\text { DP. } \\
\text { floración }\end{array}$ \\
\hline Altura $(\mathrm{cm})$ & 1 & & & & & & & & & & & \\
\hline $\begin{array}{l}\text { Diámetro } \\
(\mathrm{mm})\end{array}$ & $0.675^{*}$ & 1 & & & & & & & & & & \\
\hline Núm. hojas & 0.042 & 0.103 & 1 & & & & & & & & & \\
\hline Núm. ramas & $0.333^{*}$ & 0.18 & $0.318^{*}$ & 1 & & & & & & & & \\
\hline Núm. flores & 0.134 & 0.088 & 0.166 & -0.104 & 1 & & & & & & & \\
\hline Núm. frutos & 0.284 & $0.344^{*}$ & -0.226 & 0.025 & $0.364^{*}$ & 1 & & & & & & \\
\hline L. hoja (cm) & $0.368^{*}$ & $0.382^{*}$ & $-0.422^{*}$ & $-0.363^{*}$ & 0.073 & 0.106 & 1 & & & & & \\
\hline A. hoja $(\mathrm{cm})$ & $0.327^{*}$ & $0.454^{* *}$ & -0.285 & -0.22 & $0.324^{*}$ & 0.175 & $0.857^{*}$ & 1 & & & & \\
\hline $\begin{array}{l}\text { L. peciolo } \\
(\mathrm{cm})\end{array}$ & 0.187 & 0.24 & $-0.472^{*}$ & $-0.484^{*}$ & 0.207 & 0.109 & $0.851^{*}$ & $0.686^{*}$ & 1 & & & \\
\hline $\begin{array}{l}\text { L. foliolo } \\
(\mathrm{mm})\end{array}$ & 0.249 & $0.386^{*}$ & $-0.47^{*}$ & -0.048 & -0.088 & 0.24 & 0.288 & 0.219 & $0.304^{*}$ & 1 & & \\
\hline $\begin{array}{l}\text { A. foliolo } \\
(\mathrm{mm})\end{array}$ & -0.085 & 0.045 & -0.187 & -0.117 & 0.084 & 0.269 & 0.068 & 0.017 & 0.126 & $0.636^{*}$ & 1 & \\
\hline DP. floración & -0.161 & -0.134 & -0.127 & -0.113 & $-0.599^{* *}$ & $-0.361^{*}$ & -0.102 & -0.199 & -0.219 & 0.078 & $-0.343^{*}$ & 1 \\
\hline
\end{tabular}

${ }^{* *}=$ correlación significativa a $0.01 .{ }^{*}=$ correlación significativa a 0.05 . 


\section{Análisis de componentes principales}

El análisis de componentes principales demostró la variabilidad en los caracteres morfológicos en las 20 accesiones de moringa (Cuadro 6). El porcentaje de variación fue de 52.87, 37.54, 6.16, 1.98 y 0.63 para los componentes $1,2,3,4$ y 5 , respectivamente.

Cuadro 6. Análisis de componentes principales para descriptores morfológicos de Moringa oleífera a los 301 DDT.

\begin{tabular}{|c|c|c|c|c|c|c|}
\hline Núm. & Descriptor & $\mathrm{CP} 1$ & CP 2 & CP 3 & $\mathrm{CP} 4$ & CP 5 \\
\hline 1 & Altura $(\mathrm{cm})$ & 0.6 & 0.785 & 0.029 & -0.11 & -0.094 \\
\hline 2 & Diámetro (mm) & 0.075 & 0.091 & 0.006 & 0.291 & 0.856 \\
\hline 3 & Número de hojas & 0.04 & -0.025 & 0.906 & 0.378 & -0.09 \\
\hline 4 & Ramas & 0.026 & 0.023 & 0.113 & -0.157 & 0.179 \\
\hline 5 & Número de flores & 0.013 & -0.009 & 0.003 & 0.025 & -0.03 \\
\hline 6 & Número de frutos & 0.006 & 0 & -0.01 & -0.009 & 0.038 \\
\hline 7 & Longitud de la hoja (cm) & 0.042 & 0.046 & -0.264 & 0.458 & -0.238 \\
\hline 8 & Ancho de la hoja (cm) & 0.064 & 0.036 & -0.276 & 0.713 & -0.102 \\
\hline 9 & Longitud del peciolo $(\mathrm{cm})$ & 0.013 & 0.002 & -0.093 & 0.114 & -0.062 \\
\hline 10 & Longitud del foliolo (mm) & 0.006 & 0.024 & -0.099 & -0.023 & 0.339 \\
\hline 11 & Ancho del foliolo (último) (mm) & 0.008 & -0.014 & -0.026 & -0.019 & 0.115 \\
\hline 12 & Hojas & 0 & 0 & 0 & 0 & 0 \\
\hline 13 & Color de peciolo de la hoja & 0.005 & 0.009 & -0.001 & 0.014 & 0.067 \\
\hline 14 & Forma de la hoja & 0 & 0 & 0 & 0 & 0 \\
\hline 15 & Ápice de la hoja (en hoja madura) & 0 & 0 & 0 & 0 & 0 \\
\hline 16 & Pubescencia en la hoja & 0.004 & -0.003 & 0.001 & -0.011 & -0.029 \\
\hline 17 & Días a la primera floración & -0.791 & 0.609 & 0.034 & 0.043 & -0.006 \\
\hline 18 & Color de la flor & 0.008 & 0.001 & -0.004 & 0.015 & 0.103 \\
\hline 19 & Manchas purpuras en las flores & -0.003 & 0.008 & 0.003 & 0.007 & 0.016 \\
\hline \multirow[t]{3}{*}{20} & Color de anteras & 0.002 & -0.002 & -0.003 & -0.007 & 0.027 \\
\hline & Eigenvalue & 938.953 & 666.755 & 109.553 & 35.323 & 11.289 \\
\hline & Variance $(\%)$ & 52.874 & 37.546 & 6.169 & 1.989 & 0.636 \\
\hline
\end{tabular}

$\mathrm{CP}=$ componente principal.

En la Figura 3, bidimensional de los análisis de componentes principales 1 y 2 se observan 3 grupos. El grupo A comprende las accesiones O2, Y2, C2, G2, C3, V2, G4, C1, V3, Y1, O4 y V1. El grupo B comprende las accesiones G3, O3, G1, Y3 y C4. En el grupo C se identificaron las poblaciones V4, O1 y Y4 (Figura 3). La agrupación fue resultado del CPA con base en el análisis de los descriptores morfológicos. 


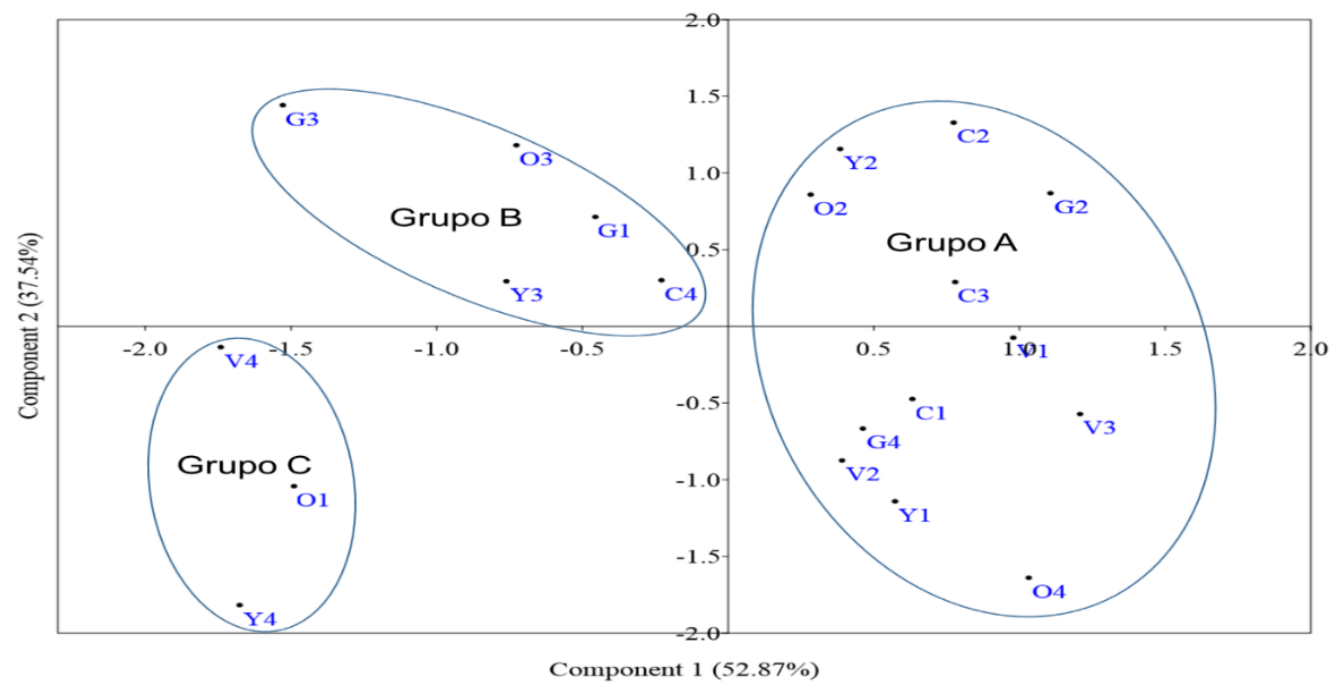

Figura 3. Dispersión de los componentes 1 y 2 para las 20 accesiones de Moringa oleifera Lam., del sur-sureste de México.

\section{Clúster}

En el grupo A se formaron dos subgrupos: I (O2, Y2, C2, G2) y II (O4, G4, C1, Y1, V2, V3, C3 y V1). En el grupo B se formaron los subgrupos: I (O3, G1, C4, G3) y II (Y3). En el grupo C se identificaron a las accesiones V4, O1 y Y4 (Figura 4).

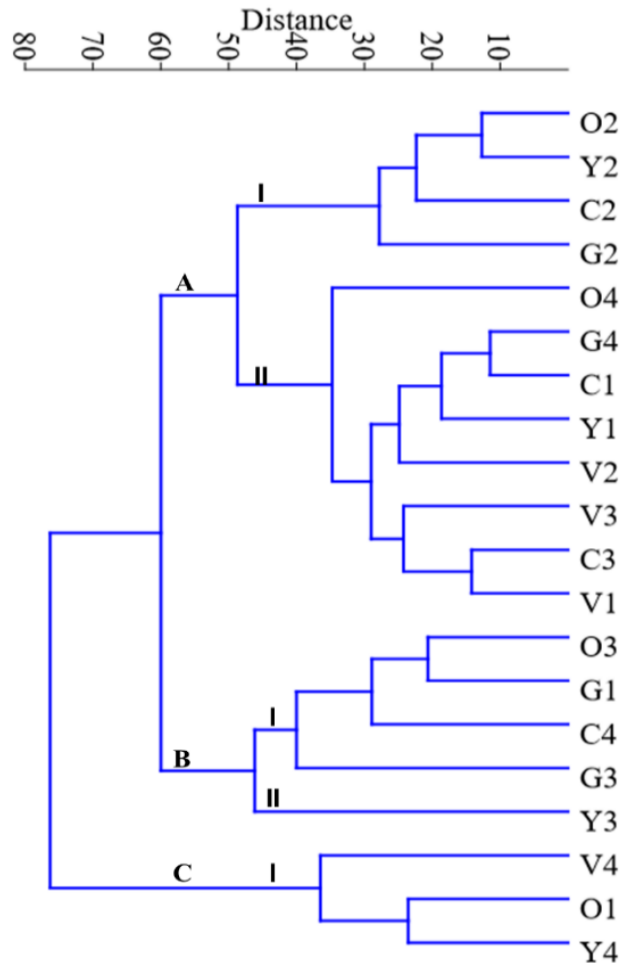

Figura 4. Análisis de clúster basado en datos morfológicos de 20 accesiones de Moringa oleifera recolectadas en el sur-sureste de México. 


\section{Conclusiones}

Existe una diversidad morfológica en los accesos de moringa provenientes del sur-sureste de México. Esta diversidad puede servir para reforzar el conocimiento de moringa y ampliar la información respecto a su fisiología, fenología y producción. El conocimiento morfológico de las accesiones permitirá la creación de programas de conservación, selección y generación de materiales élite con mayor potencial adaptativo, resistencia a plagas y enfermedades y con mayor capacidad productiva y contenido nutricional.

\section{Literatura citada}

Baiyeri, K. P.; Apeh, P.; Stevens, G. C.; Ndukwe, O.; Aba, S. C. and Otitoju, G. T. 2015. Growth performance and nutrient quality of three Moringa oleifera accessions grown as potplant under varied manure rates and watering intervals. Afr. J. Biotechnol. 14(24):1996-2004. doi: 10.5897/AJB2014.14359.

Bradshaw, J. H. D. and Schemske, D. W. 2003. Allele substitution at a flower colour locus produces a pollinator shift in monkeyflowers. Nature. 426(6963):176-178. doi: 10.1038/nature02106.

Chaves-Bedoya, G.; Galvis-Pérez, Z. L. y Ortiz-Rojas, L. Y. 2017. Diversidad genética de Moringa oleifera Lam. En el nororiente colombiano utilizando marcadores RAMs. Rev. Colomb. Cienc. Hortíc. 11(2):408-415. doi: http://dx.doi.org/10.17584/rcch.2017v11i2.7343.

Dao, M. C. E. and Kabore, K. H. 2015. Morphological characteristic variation of eleven provenances of Moringa oleifera seedlings grown in the northern sudanese area of burkina faso. Afr. J. Plant Sci. 9(10):401-411. doi: 10.5897/AJPS2015.1334.

Du-Toit, E. S.; Fotouo, H. and Robbertse, P. J. 2017. Seed storage conditions influence germination of Moringa oleifera Lam. seed. Acta Hortic. 1158:441-446. https://www.cabdirect.org/ cabdirect/abstract/20173287864.

Folkard, G.; Sutherland, J. and Shaw, R. 1999. Water clarification using Moringa oleifera coagulant. 'Water and environmental health at london and loughborough' (well), Loughborough University, Loughborough. 109-112 pp.

Förster, N.; Ulrichs, C.; Schreiner, M.; Arndt, N.; Schmidt, R. and Mewis, I. 2015. Ecotype variability in growth and secondary metabolite profile in Moringa oleifera: impact of sulfur and water availability. J. Agric. Food Chem. 63(11):2852-2861. doi: 10.1021/jf506174v.

Fotouo-M, H.; Du-Toit, E. S. and Robbertse, P. J. 2015. Germination and ultrastructural studies of seeds produced by a fast-growing, drought-resistant tree: implications for its domestication and seed storage. AoB Plants. 1-12. doi: 10.1093/aobpla/plv016.

Kumar, A. P.; Sarawgi, A. K.; Bhandarkar, S. and Ojha, G. C. 2017. Agro-morphological characterization and morphological based genetic diversity analysis of Rice (Oryza sativa L.) germplasm. J. Pharmacognosy Phytochem. 6(6):75-80. http://www.phytojournal.com/ archives/?year $=2017 \& \mathrm{vol}=6 \&$ issue $=6 \&$ ArticleId $=2062$.

Ledea-Rodríguez, J. L. G.; Rosell-Alonso, D. G.; Benítez-Jiménez, R. C.; Arias-Pérez, J. V.; RayRamírez, and J. J. y Reyes-Pérez. 2018. Producción de semillas de variedades de Moringa oleifera Lam. En el valle del cauto. Agron. Mesoam. 29(2):415-423. http://dx.doi.org/ 10.15517/ma.v29i2.29545.

Leone, A.; Spada, A.; Battezzati, A.; Schiraldi, A.; Aristil, J. and Bertoli, S. 2015. Cultivation, genetic, ethnopharmacology, phytochemistry and pharmacology of Moringa oleifera leaves: an overview. Inter. J. Mol. Sci. 16(6):12791-12835. Doi: 10.3390/ijms160612791. 
Martín, C.; Martín, G.; García, A.; Fernández, T.; Hernández, E. and Puls, J. 2013. Potential applications of Moringa oleifera. A critical review. Pastos y Forrajes. 36(2):137-149. https://www.researchgate.net/publication/303986314.

Mgendi, M. G.; Nyomora, A. M. and Manoko, M. K. 2011. Using morphological markers to assess variations between and within cultivated and non-cultivated provenances of Moringa oleifera Lam. in Tanzania. J. Life Sci. 5:387-392.

NRC. 2006. National Research Council. 'moringa' -lost crops of Africa National Academic Press. ISBN: 978-0-309-10333-6. 247-267 pp.

Olguín, C. P. 1999. Fertiirrigación orgánica: investigación y transferencia. Terra Latinoam. 17(3):175-178. https://www.redalyc.org/pdf/573/57317301.pdf.

Opare-Obuobi, K. 2012. Characterisation of local and exotic accessions of moringa (Moringa oleifera Lamarck). Department of crop science. College of Agriculture and Consumer Sciences University of Ghana $132 \mathrm{p}$.

Panshin, A. J. and Zeeuw, C. D. 1970. Textbook of wood technology. Volume I. $3^{\text {ra }}$ (Ed.). Structure, identification, uses, and properties of the commercial woods of the United States and Canada. Textbook of wood technology. Volume I. Structure, identification, uses, and properties of the commercial woods of the United States and Canada. 63-64 pp.

Pérez, A.; Sánchez, T.; Armengol, N. y Reyes, F. 2010. Características y potencialidades de Moringa oleifera, Lamark: una alternativa para la alimentación animal. Pastos y Forrajes. 33(4):1-16.

Popoola, J. O.; Bello, O. A. and Obembe, O. O. 2016. Phenotypic intraspecific variability among some accessions of Drumstick (Moringa oleifera Lam.). Can. J. Pure Appl. Sci. 10(1):3681-3693. https://www.researchgate.net/publication/289521612.

PPV and FR. 2001. Guidelines for the conduct of test for distinctiveness, uniformity and stability on drumstick (Moringa oleifera Lam). Protection of plant varieties and farmers' rights authority. government of India. New Delhi, India. 1-29 pp.

Price, L. M. 2000. The Moringa tree: revised in 2000 by Kristin Davis. 1-14 pp.

Ramos, L. M.; Costa, R. S.; Môro, F. V. and Silva, R. C. 2010. Morfología de frutos e sementes e morfofunção de plântulas de Moringa (Moringa oleifera Lam.). Comunicata Scientiae. 1(2):156-160. https://dialnet.unirioja.es/servlet/articulo?codigo=6294707.

Resmi, D. S.; Celine, V. A. and Rajamon, L. 2005. Variability among drumstick (Moringa oleifera Lam.) accessions from Central and Southern Kerala. J. Tropical Agric. 43(1-2):83-85. http://jtropag.kau.in/index.php/ojs2/article/view/141.

Reverté, S.; Retana, J.; Gómez, J. M. and Bosch, J. 2016. Pollinators show flower colour preferences but flowers with similar colours do not attract similar pollinators. Ann. Bot. 118(2):249-257. Doi: 10.1093/aob/mcw103.

Santiago, M. T. B. and Bezerra, N. E. 2017. Ecophysiology of Moringa oleifera Lam. in function of different rainfall conditions. Rev. Geama. 3(4):236-241.

Taiz, L. y Zeiger, E. 2009. Fisiologia vegetal. $4^{\text {ta. }}$ (Ed.). Porto Alegre. Universidad Jaume. Artmed. $656 \mathrm{p}$.

Tesfay, S. Z.; Bertling, I.; Odindo, A. O.; Workneh, T. S. and Mathaba, N. 2011. Levels of antioxidants in different parts of moringa (Moringa oleifera) seedling. Afr. J. Agric. Res. 6(22):5123-5132. Doi: https://doi.org/10.5897/AJAR11.1101.

Valdés, R. O. A.; Wassenaar, O. M. P.; Ruiz R. y Pérez, A. V. 2014. Potencial de la asociación moringa y ricinus en el subtrópico veracruzano. Rev. Mex. Cienc. Agríc. 9(2014):16731686. http://cienciasagricolas.inifap.gob.mx/index.php/agricolas/article/view/1056. 
Vasconcelos, M. C.; Costa, J. C.; Sousa, J. P. S.; Santana, F. V.; Soares, T. F. S. N.; Oliveira L. F. G. D. and Silva-Mann, R. 2019. Biometric and physiological responses to water restriction in Moringa oleifera seedlings. Floresta e Ambiente. 26(1):1-8. Doi: https://doi.org/ 10.1590/2179-8087.016515.

Zaku, S. G.; Emmanuel, S.; Tukur, A. A. and Kabir, A. 2015. Moringa oleifera: an underutilized tree in Nigeria with amazing versatility: A review. Afr. J. Food Sci. 9(9):456-461. https://doi.org/10.5897/AJFS2015.1346. 